M. Hochster

Nagoya Math. J.

Vol. 51 (1973), 25-43

\title{
CONTRACTED IDEALS FROM INTEGRAL EXTENSIONS OF REGULAR RINGS
}

\author{
M. HOCHSTER ${ }^{1}$
}

0. Introduction. The purpose of this paper is to consider the following question: if $R$ is a regular Noetherian ring and $S \supset R$ is a module-finite $R$-algebra, is $R$ a direct summand of $S$ as $R$-modules? An affirmative answer is given if $R$ contains a field, and it is shown that if the completions of the local rings of $S$ possess maximal CohenMacaulay modules in the sense of $\S 1$ of [6] then the conclusion is valid in this case too. Hence, if Conjecture $E$ of [6] is true then the question raised here has an affirmative answer without further restriction on the regular Noetherian ring $R$, and it will be shown here that only a greatly weakened version of Conjecture $E$ is needed.

It follows from our results on direct summands that if $R$ contains a field, its local rings are regular, and $S$ is an extension algebra integral over $R$, then every ideal of $R$ is contracted, i.e. if $I \subset R$ then $I S \cap R=I$. In fact, we prove the direct summand result by proving first that certain key ideals $I$ of a regular local ring have this property. In the final section of the paper we consider briefly some propositions about the class of domains such that every ideal is contracted from every integral extension.

Throughout this paper, all rings are commutative, with identity, all modules are unitary, and ring homomorphisms are assumed to preserve the identity.

1. Regular rings. Our first reductions in the problem are consequences of the following:

LEMMA 1. Let $R \subset S$ be rings and assume that $S$ is finitely presented as an $R$-module. Then $R$ is a direct summand of $S$ if and only if for

Received November 6, 1972.

1 This research was partially supported by NSF grant GP-29224X. 
each maximal ideal $P$ of $R, R_{p}$ is a direct summand of $S_{p}$.

Moreover, if $T$ is a faithfully flat $R$-algebra then $R$ is a direct summand of $S$ if and only if $T=R \otimes_{R} T$ is a direct summand of $S \otimes_{R} T$.

Proof. Consider the exact sequence

$$
0 \rightarrow R \rightarrow S \rightarrow S / R \rightarrow 0 .
$$

To ask whether $R$ is a direct summand of $S$ is the same as to ask whether

$$
\operatorname{Hom}_{R}(S / R, S) \rightarrow \operatorname{Hom}_{R}(S / R, S / R)
$$

is surjective. This map is surjective if and only if for each maximal ideal $P$ of $R$,

$$
\operatorname{Hom}_{R}(S / R, S) \otimes_{R} R_{P} \rightarrow \operatorname{Hom}_{R}(S / R, S / R) \otimes_{R} R_{P}
$$

is surjective, and since $S$ (and hence $S / R$ ) is finitely presented, we have the commutative diagram:

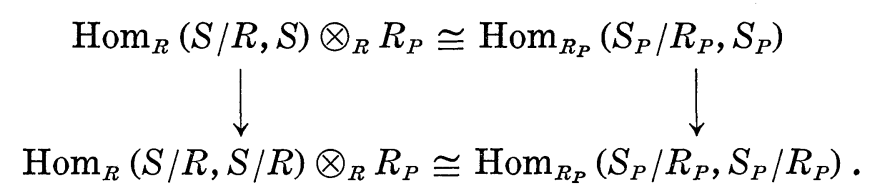

The first part of the theorem follows at once.

For the second part we note that since $T$ is faithfully flat

$$
\operatorname{Hom}_{R}(S / R, S) \rightarrow \operatorname{Hom}_{R}(S / R, S / R)
$$

is surjective if and only if

$$
\operatorname{Hom}_{R}(S / R, S) \otimes_{R} T \rightarrow \operatorname{Hom}_{R}(S / R, S / R) \otimes_{R} T
$$

is surjective, and since $S, S / R$ are finitely presented and $T$ is flat we have the commutative diagram:

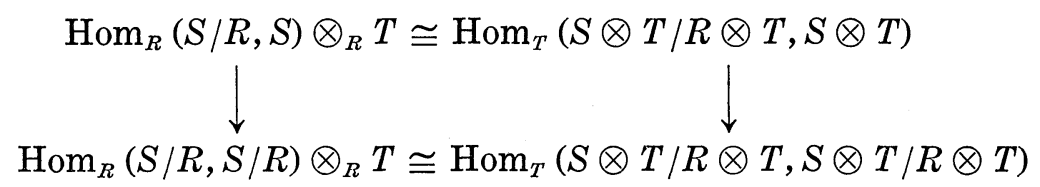

and so the second part follows.

Remark 1. The criteria of Lemma 1 apply to determine whether 
an arbitrary short exact sequence of finitely presented $R$-modules is split, not just

$$
0 \rightarrow R \rightarrow S \rightarrow S / R \rightarrow 0 .
$$

This is evident from the proof.

Proposition 1. If $R \subset S$ are rings such that $R$ is a direct summand of $S$ then for each ideal $I$ of $R, I S \cap R=I$.

Proof. See [7], Proposition 10.

The condition that $R$ be a direct summand of an extension ring $S$ is equivalent, in the terminology of [7], to the existence of a Reynolds operator from $S$ to $R$. The consequences of the existence of a Reynolds operator are discussed in detail in [7] Propositions 9-12. Such operators occur frequently in invariant theory over fields of characteristic zero.

We note that if $R$ is a domain which is a direct summand of every module-finite extension, then $R$ is integrally closed. For if $b, a \neq 0$ are in $R$ and $b / a$ is integral over $R$, then since $R[b / a]$ is module-finite over $R$ we have $b \in(a R[b / a]) \cap R=a R$ and $b / a \in R$.

The following two results show that if $R$ contains the rationals integral closure is also sufficient. The next result is well known but we include a short proof for the sake of completeness.

LEMMA 2. If $R$ is an integrally closed domain which contains the rationals $Q$ and $S$ is an integral extension domain of $R$ of finite degree, then $R$ is a direct summand of $S$.

Proof. There is no harm in enlarging $S$. Hence, we might as well assume that $S$ is the integral closure of $R$ in a finite normal field extension $L$ of the fraction field $K$ of $R$. Let $d=[L: K]$. Then $(1 / d) \operatorname{Tr}_{L / K}$ retracts $S$ onto $R$. In fact, if $r \in R$ (or $K$ ) $\operatorname{Tr}_{L / K}(r)=d r$. On the other hand, the trace of an integral element is integral and every element of $K$ integral over $R$ is in $R$.

Lemma 2 shows that the hypothesis of regularity is much more than sufficient in case $R$ contains the rationals. To dispel the idea that anything much weaker than regularity might be enough in characteristic $p$ we give an example.

EXAMPLE 1. Let $K$ be an algebraically closed field of characteristic 2. Let $u, v, x, t$, and $z$ be indeterminates. Let $D=K\left[u^{2}, v^{2}, u^{3}+v^{3}\right] \subset$ 
$K[u, v]$. The $K$-homomorphism from $K[x, y, z]$ onto $D$ which takes $x, y, z$ to $u^{2}, v^{2}, u^{3}+v^{3}$, respectively, has as its kernel the principal ideal $\left(x^{3}+\right.$ $y^{3}+z^{2}$ ), so that $D$ is Gorenstein (and, in fact, a complete intersection), and it is easy to see that $D$ is integrally closed in its fraction field. In $D, u^{3}+v^{3} \notin\left(u^{2}, v^{2}\right) D$, but in $K[u, v]$, which is integral over $D, u^{3}+v^{3} \in$ $\left(u^{2}, v^{2}\right) K[u, v]$. Thus, $\left(u^{2}, v^{2}\right)$ is not contracted from $K[u, v]$, and it follows that $D$ is not a direct summand of its integral extension $K[u, v]$, by Proposition 1. The essential properties of this example are preserved by localizing at the maximal ideals $((u, v) \cap D$ and $(u, v))$ and even by, furthermore, completing the resulting local rings. Hence, even an analytically normal Gorenstein algebro-geometric local ring fails to be a summand of all of its module-finite extensions in characteristic $p$. The author has not found any natural condition weaker than regularity which suffices (although regularity is not necessary).

The next lemma shows that we only need to look at domain extensions.

LEMMA 3. Let $R$ be a domain and $S$ an integral extension of $R$. Then there is a prime $P$ of $S$ such that $P \cap R=(0)$, and if $R$ is a direct summand of $S / P$ (under the induced inclusion), then $R$ is a direct summand of $S$.

Proof. Since $R-\{0\}$ is a multiplicative system in $S$ we can take $P$ to be any ideal of $S$ maximal with respect to disjointness from $R-\{0\}$. We have a commutative diagram:

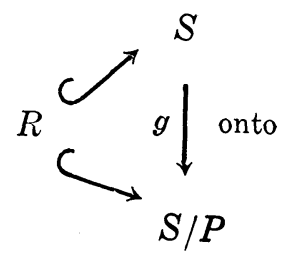

and is follows that if $f: S / P \rightarrow R$ is an $R$-module retraction then $f g$ : $S \rightarrow R$ is an $R$-module retraction.

Suppose we are given, now, a regular Noetherian ring $R$ and a module-finite $R$-algebra $S \supset R$, and we want to decide whether $R$ is a direct summand of $S$. By using the first part of Lemma 1 we can reduce to the case where $R$ is local, and by using the second part to the case where $R$ is complete, taking $T=\hat{R}$, the completion of $R$. Using Lemma 3 we can reduce to the case where $S$ is a domain. However, for the moment, we shall not make all of these reductions but we give 
instead a criterion for $R$ to be a direct summand of $S$ when $R$ is a regular local ring and $S$ is module-finite over $R$.

THEOREm 1. Let $(R, P)$ be a regular local ring and let $x_{1}, \cdots, x_{n}$ be a regular system of parameters for $R$ (i.e. $\operatorname{dim} R=n$ and $P=\left(x_{1}, \cdots, x_{n}\right) R$ ). Let $R \subset S$, where $S$ is a module-finite $R$-algebra. Then $R$ is a direct summand of $S$ if and only if for every positive integer $k$,

$$
\left(x_{1} \cdots x_{n}\right)^{k} \notin\left(x_{1}{ }^{k+1}, \cdots, x_{n}{ }^{k+1}\right) S
$$

Proof. Let $I_{k}=\left(x_{1}^{k}, \cdots, x_{n}^{k}\right) R$. Since $R$ is regular and $x_{1}^{k}, \cdots, x_{n}^{k}$ is a system of parameters, $R_{k}=R / I_{k}$ is Gorenstein and zero-dimensional: let $P_{k}=P / I_{k}$. Then $\mathrm{Ann}_{R_{k}} P_{k}$ will be isomorphic to a single copy of $R / P$, and every nonzero ideal of $R_{k}$ will contain $\operatorname{Ann}_{R_{k}} P_{k}$. It is quite easy to see, in fact, that $\mathrm{Ann}_{R_{k}} P_{k}$ is generated by the residue class modulo $I_{k}$ of $u_{k}=\left(x_{1} \cdots x_{n}\right)^{k-1}$. It follows that every ideal of $R$ strictly larger than $I_{k}$ contains $u_{k}$.

Now, if $R$ is a direct summand of $S$ then every ideal of $R$ will be contracted. Since $u_{k+1} \notin I_{k+1} R$, we will then have $u_{k+1} \notin I_{k+1} S$, which is precisely the condition asserted in Theorem 1 .

To prove the converse, assume that $u_{k+1} \notin I_{k+1} S$, all $k$. Let $\hat{R}$ be the completion of $R$. By the second statement of Lemma 1 , it suffices to show that $\hat{R}$ is a direct summand of $S \otimes_{R} \hat{R}$, which is a module-finite extension algebra of $\hat{R}$. Since $\hat{R}$ is faithfully flat over $R, S \otimes_{R} \hat{R}$ is faithfully flat over $S$. Then $u_{k+1} \notin\left(I_{k+1}\left(S \otimes_{R} \hat{R}\right)\right) \cap \hat{R}$, because if it did, we would have $u_{k+1} \in\left(\left(I_{k+1}\left(S \otimes_{R} \hat{R}\right)\right) \cap S\right) \cap \hat{R}$ and since $S \otimes_{R} \hat{R}$ is faithfully flat over $S,\left(I_{k+1}\left(S \otimes_{R} \hat{R}\right)\right) \cap S=I_{k+1} S$ and $u_{k+1} \in\left(I_{k+1} S \cap R\right) \cap R=$ $\left(I_{k+1} S\right) \cap R$, contradiction. Hence, we may assume without loss of generality that $R$ is complete.

Since the ideals $I_{k}$ are cofinal with the powers of $P$ and $R$ is complete,

$$
\operatorname{Hom}_{R}(S, R)=\lim \operatorname{Hom}_{R}\left(S_{k}, R_{k}\right)
$$

where $S_{k}=S / I_{k} S$. We also note the isomorphisms

$$
\operatorname{Hom}_{R}\left(S_{k}, R_{k}\right) \cong \operatorname{Hom}_{R_{k}}\left(S_{k}, R_{k}\right) \text {. }
$$

Now, $I_{k} S \cap R=I_{k}$, because if $J=I_{k} S \cap R$ and $J \supsetneq I_{k}$, then $u_{k} \in J$, and this contradicts our hypothesis. Hence, the inclusion $R \rightarrow S$ induces an inclusion $R_{k} \rightarrow S_{k}$ for each $k$. Since $R_{k}$ is a zero-dimensional Gorenstein 
ring, it is injective as an $R_{k}$-module, and it follows that for each $k$, the inclusion $R_{k} \rightarrow S_{k}$ splits, i.e. $R_{k}$ is a direct summand of $S_{k}$. For each $k$, let $h_{k}: \operatorname{Hom}_{R}\left(S_{k}, R_{k}\right) \rightarrow R_{k}$ by $h_{k}(\phi)=\phi\left(1_{k}\right)$ where $1_{k} \in R_{k} \subset S_{k}$ is the image of $1 \in R \subset S$, and let $h: \operatorname{Hom}_{R}(S, R) \rightarrow R$ by $h(\phi)=\phi(1)$. Then $H_{k}=h_{k}^{-1}(1)$ (respectively, $H=h^{-1}(1)$ ) is the set of splittings of $R_{k} \rightarrow S_{k}$ (respectively, $R \rightarrow S$ ) and the inverse limit relation (*) induces

$$
H=\lim _{\leftarrow k} H_{k}
$$

Here $H_{k}$ (respectively, $H$ ) is a coset (or translate) of a submodule of $\operatorname{Hom}_{R}\left(S, R_{k}\right)$ (respectively, $\operatorname{Hom}_{R}(S, R)$ ) and the maps are restricted module homomorphisms. All we need to show to complete the proof is that $H \neq \phi$. Since each $R_{k}$ is a summand of $S_{k}$, each $H_{k} \neq \phi$. But an inverse limit of nonempty cosets in Artinian modules is nonempty. To see this, note that for each $k$ the decreasing sequence of nonempty subcosets $\operatorname{Im}\left(H_{i+k} \rightarrow H_{k}\right)$ of $H_{k}$ stabilizes, since their lengths must stabilize. Denote this subcoset of $H_{k}$ by $E_{k}$. Then the $E_{k}$ form a subsystem of nonempty subcosets and surjective maps so that

$$
\phi \neq \lim _{\rightarrow k} E \subset H .
$$

This completes the proof.

Remark 2. Theorem 1 generalizes in various ways. We indicate a number of these generalizations. First, it is not necessary to assume that $S$ is an $R$-algebra. Assume that $R$ is regular, as before, let $x_{1}, \cdots, x_{n}$ be as before, and let $E$ be a finitely generated $R$-module. Let $e \in E$ be such that $\mathrm{Ann}_{R} e=(0)$, i.e. $R \rightarrow E$ by $r \mapsto r e$ is a monomorphism. Then $R e$ is a direct summand of $E$ if and only if for every integer $k \geq 0$,

$$
\left(x_{1} \cdots x_{n}\right)^{k} e \notin\left(x_{1}^{k+1}, \cdots, x_{n}^{k+1}\right) E .
$$

The proof is essentially the same.

Moreover, it is not necessary to assume that $R$ is regular. Let $(R, P)$ be any local ring, and let $\left\{I_{k}\right\}$ be a sequence of irreducible $P$-primary ideals cofinal with the powers of $P$. (If $R$ is Gorenstein and $x_{1}, \cdots, x_{n}$ is any system of parameters, we can take $I_{k}$ to be $\left(x_{1}^{k}, \cdots, x_{n}^{k}\right)$.) Let $u_{k} \in R$ generate the minimum nonzero ideal of $R / I_{k}$ modulo $I_{k}$. (Note that a $P$-primary ideal $I$ is irreducible if and only if $R / I$ is a 0 -dimensional Gorenstein ring.) Let $E$ be a finitely generated $R$-module and $e \in E$ be 
such that $\mathrm{Ann}_{R} e=(0)$. Then Re is a direct summand of $E$ if and only if for every integer $k \geq 0$.

$$
u_{k} e \notin I_{k} E \text {. }
$$

(If $R$ is Gorenstein, $x_{1}, \cdots, x_{n}$ is a system of parameters, and $I_{k}=$ $\left(x_{1}^{k}, \cdots, x_{n}^{k}\right)$, we may always take $\left.u_{k+1}=\left(x_{1} \cdots x_{n}\right)^{k} u_{1}\right)$. The proof of this result follows precisely the lines of the proof of Theorem 1 .

Remark 3. We also note the following result. Let $R$ be a regular local ring and $x_{1}, \cdots, x_{n}$ a regular system of parameters. Let $E$ be a finitely generated $R$-module. Then $E$ has a direct summand isomorphic to $R$ if and only if $\mathrm{Ann}_{R} E=0$ and for every integer $k \geq 0$,

$$
\left(x_{1} \cdots x_{n}\right)^{k} E \not \subset\left(x_{1}^{k+1}, \cdots, x_{n}^{k+1}\right) E .
$$

To see why this is true let

$$
E_{k}=\left(x_{1}^{k+1}, \cdots, x_{n}^{k+1}\right) E:\left(x_{1} \cdots x_{n}\right)^{k} R \subset E .
$$

Then $\left\{E_{k}\right\}$ is an increasing sequence of submodules of $E$ and we can choose $k$ such that $E_{k}=E_{k+1}=\cdots$. Then $E_{k} \neq E$. Also, if $T=$

$$
\{e \in E: \text { for some } r \in R-\{0\}, r e=0\}
$$

then $T$ is a proper submodule of $E$. Hence, $E \neq E_{k} \cup T$, and we can choose $e$ not in $E_{k}$ or $T$. Then $R e \cong R$ is a direct summand of $E$, by the first part of Remark 2.

We now are ready to handle the "geometric case."

THEOREM 2. Let $R$ be a ring which contains a field and suppose that for every prime $P$ of $R, R_{P}$ is a regular local ring. Let $S \supset R$ be an $R$-algebra which is finitely presented as an $R$-module. Then $R$ is a direct summand of $S$.

Hence, if $R$ is a regular Noetherian ring which contains a field and $S$ is a module-finite extension algebra, then $R$ is a direct summand of $S$.

Proof. We note that the second statement follows from the first simply because a finitely generated module over a Noetherian ring is finitely presented.

As for the proof of the first statement, we first handle the trivial case where $R$ contains a field of characteristic zero. By Lemma 1, we 
can assume that $R$ is local, by Lemma 3 we can assume that $S$ is a domain, and the result follows at once from Lemma 2.

Now assume that $R$ contains a field of characteristic $p>0$, so that for every $t$ the map $h_{t}: S \rightarrow S$ by $h_{t}(r)=r^{\left(p^{t}\right)}$ is a ring homomorphism. As above, we can assume that $R$ is local, and from the second part of Lemma 1 we can assume that $R$ is complete. From Lemma 3 we can also assume that $S$ is a domain. Thus, $S$ will also be a complete local domain (module-finite over $R$ ). Let $x_{1}, \cdots, x_{n}$ be a regular system of parameters for $R$. To complete the proof we need only show that there is no relation of the form

$$
\left(x_{1} \cdots x_{k}\right)^{k}=\sum_{i=1}^{n} s_{i} x_{i}^{k+1},
$$

where the $s_{i} \in S$, by Theorem 1 . We shall give two different proofs that (\#) is impossible. The first is more elementary but the second yields somewhat more information.

For the first proof, we note that since $S$ is a domain, it is torsionfree over $R$, and consequently can be embedded in a free $R$-module. Hence, there is an $R$-homomorphism $g: S \rightarrow R$ such that $a=g(1) \neq 0$. It follows that we can choose $t$ so large that $a \notin P^{p^{t}}$. We can raise both sides of (\#) to the $p^{t}$ power to obtain:

$$
\left(x_{1} \cdots x_{n}\right)^{k p^{t}} \cdot 1=\sum_{i=1}^{n} s_{i}^{p^{t}} x_{i}^{k p^{t}+p^{t}}
$$

Apply $g$ to both sides:

$$
\left(x_{1} \cdots x_{n}\right)^{k^{\prime}} a=\sum_{i=1}^{n} r_{i} x_{i}^{k^{\prime}+p^{t}}
$$

where $k^{\prime}=k p^{t}$ and $r_{i}=g\left(s_{i}{ }^{t}\right) \in R$. Hence, in the regular ring $R$,

$$
a \in\left(x_{1}^{k^{\prime}+p^{t}}, \cdots, x_{n}^{k^{\prime}+p^{t}}\right):\left(x_{1} \cdots x_{n}\right)^{k^{\prime}}
$$

and since $x_{1}, \cdots, x_{n}$ is an $R$-sequence it follows that this colon of ideals can be computed precisely as though $x_{1}, \cdots, x_{n}$ were indeterminates over $Z$ (see [3] or [9]), so that

$$
a \in\left(x_{1}^{p^{t}}, \cdots, x_{n}^{p^{t}}\right) \subset P^{p^{t}},
$$

a contradiction. This completes the proof of Theorem 2.

Before giving the second proof of the impossibility of (\#), we discuss 
briefly the problem of proving Theorem 2 without the hypothesis that $R$ contains a field. We reduce at once to the case where $R$ is a complete local ring and $S$ is a domain module-finite over $R$, and all we need to show is that (\#) cannot hold. Since $x_{1}, \cdots, x_{n}$ is a system of parameters of $S$ ( $S$ is local because $R$ is complete) all we need is the following:

CONJECTURE 1. If $S$ is any local ring and $x_{1}, \cdots, x_{n}$ is a system of parameters for $X$, then for every integer $k \geq 0$,

$$
\left(x_{1} \cdots x_{n}\right)^{k} \notin\left(x_{1}^{k+1}, \cdots, x_{n}^{k+1}\right) .
$$

Evidently, if we give a proof of this conjecture when $S$ contains a field of characteristic $p>0$, then we have a new way of completing the last part of the proof of Theorem 2.

Note that in Conjecture 1 , if $S$ is a counterexample, so is $\hat{S}$, its completion, and if $P$ is a prime of $\hat{S}$ of coheight $\operatorname{dim} \hat{S}=\operatorname{dim} S$, then $\hat{S} / P$ is a complete local domain which is a counterexample.

We now give a proof of Conjecture 1 when $S$ contains a field of characteristic $p>0$ which differs from the earlier argument in the proof of Theorem 2. It is based on:

Proposition 2. If $y_{1}, \cdots, y_{n}$ is a system of parameters for the local ring $(S, P)$ and $N$ is any sufficiently large positive integer, then the system of parameters $x_{1}=y_{1}^{N}, \cdots, x_{n}=y_{n}^{N}$ satisfies the conclusion of Conjecture 1.

Proof. We can assume that $S$ is complete. Then $n=\operatorname{dim} S$, and $H_{P}^{n}(S) \neq 0$, where $H_{P}^{i}$ denotes the $i$ th local cohomology module with respect to the maximal ideal $P$ of $S$. (See Part 4 of Proposition 6.4 of [4].) By Theorem 2.3 of [4], $H_{P}^{i}(S)$ can be interpreted as a direct limit of Koszul homology modules, and in the special case at hand we obtain

$$
H_{P}^{n}(S)=\lim _{\leftarrow k} S /\left(y_{1}^{k}, \cdots, y_{n}^{k}\right)
$$

where the map

$$
S /\left(y_{1}^{k}, \cdots, y_{n}^{k}\right) \rightarrow S /\left(y_{1}^{i+k}, \cdots, y_{n}^{i+k}\right)
$$

is induced by multiplication by $\left(y_{1} \cdots y_{n}\right)^{i}$. Since $H_{P}^{n}(S) \neq 0$, we can choose $N_{0}$ such that if $N \geq N_{0}$

$$
\operatorname{Im}\left(S /\left(y_{1}^{N}, \cdots, y_{n}^{N}\right) \rightarrow H_{P}^{n}(S)\right) \neq 0 .
$$


Hence, if $N \geq N_{0}$ the image of $1_{N}$ in $S /\left(y_{1}^{N+i}, \cdots, y_{n}^{N+i}\right)$ is nonzero for every $i \geq 0$, where $1_{N}$ is the residue of 1 modulo $\left(y_{1}^{N}, \ldots, y_{n}^{N}\right)$. But this says that for $N \geq N_{0}$ and every $i \geq 0$,

$$
\left(y_{1} \cdots y_{n}\right)^{i} \notin\left(y_{1}^{N+i}, \cdots, y_{n}^{N+i}\right) .
$$

In particular if $i=N k$ we have that

$$
\left(y_{1}^{N} \cdots y_{n}^{N}\right)^{k} \notin\left(\left(y_{1}^{N}\right)^{k+1}, \cdots,\left(y_{n}^{N}\right)^{k+1}\right)
$$

for all $k$ and $N \geq N_{0}$, which is just what we wanted to prove.

Thus, the nonvanishing of local cohomology in dimension $n$ is relevant to our problem.

We now give our second method for completing the proof of Theorem 2. If $S$ contains a field of characteristic $p$ and $y_{1}, \cdots, y_{n}$ is a system of parameters such that

$$
\left(y_{1} \cdots y_{n}\right)^{k}=\sum_{i=1}^{n} s_{i} y_{i}^{k+1}
$$

choose $N$ of the form $p^{t}$ as in Proposition 2. Raising both sides to the $N$ th power gives:

$$
\left(y_{1}^{N} \cdots y_{n}^{N}\right)^{k}=\sum_{i=1}^{n} s_{i}^{N}\left(y_{i}^{N}\right)^{k+1},
$$

contradicting Proposition 2.

We have shown that Conjecture 1 holds if $S$ contains a field of characteristic $p>0$. We now prove it if $S$ contains any field, by working backwards from Theorem 2.

Proposition 3. If $S$ is a local ring which contains a field and $x_{1}, \cdots, x_{n}$ is a system of parameters for $S$, then for every integer $k \geq 0$,

$$
\left(x_{1} \cdots x_{n}\right)^{k} \notin\left(x_{1}^{k+1}, \cdots, x_{n}^{k+1}\right) .
$$

Proof. We can assume without loss of generality that $S$ is a complete equicharacteristic local domain, so that $S$ is module-finite over its subring $R=K\left[\left[x_{1}, \cdots, x_{n}\right]\right]$, where $K$ is a coefficient field for $S$. Then $\left(x_{1}^{k+1}, \cdots, x_{n}^{k+1}\right) R$ is contracted from $S$, by Theorem 2 and Proposition 1 , and since $\left(x_{1} \cdots x_{n}\right)^{k} \notin\left(x_{1}^{k+1}, \cdots, x_{n}^{k+1}\right) R$, we also have that $\left(x_{1} \cdots x_{n}\right)^{k} \notin$ $\left(x_{1}^{k+1}, \cdots, x_{n}^{k+1}\right) S$, as required.

We next give a discussion of the relevance of Cohen-Macaulay modules. The following proposition is the key: 
Proposition 4. Let $S$ be a local ring and $y_{1}, \cdots, y_{n}$ a system of parameters for $S$. Suppose that there is a (possibly non-Noetherian) module $E$ over $S$ such that

1) $\left(y_{1}, \cdots, y_{n}\right) E \neq E$

2) The first Koszul homology module

$$
H_{1}\left(E ; y_{1}, \cdots, y_{n}\right)=0
$$

((2) is satisfied if $y_{1}, \cdots, y_{n}$ is an E-sequence.) Then for every integer $k \geq 1$,

$$
\left(y_{1} \cdots y_{n}\right)^{k} \notin\left(y_{1}^{k+1}, \cdots, y_{n}^{k+1}\right)
$$

Proof. Let $Z$ be the integers, let $x_{1}, \cdots, x_{n}$ be indeterminates over $Z$, let $B=Z\left[x_{1}, \cdots, x_{n}\right]$ and make $S$ into a $B$-algebra by mapping $x_{i}$ to $y_{i}, 1 \leq i \leq n$. We can think of $Z=B /\left(x_{1}, \cdots, x_{n}\right)$ as a $B$-module, and then

$$
H_{1}\left(E ; y_{1}, \cdots, y_{n}\right) \cong \operatorname{Tor}_{1}^{B}(Z, E) .
$$

Let $I=\left(x_{1}^{k}, \cdots, x_{n}^{k}\right) \subset B$ and let $J=\left(x_{1}^{k}, \cdots, x_{n}^{k},\left(x_{1} \cdots x_{n}\right)^{k+1}\right) \subset B$. It is easy to see that $J / I \cong Z$.

Let $I_{0}$ be any ideal of $B$ generated by monomials in $x_{1}, \cdots, x_{n}$ which contains a power of each $x_{i}, 1 \leq i \leq n$. We shall show that $B / I_{0}$ has a filtration in which all the factors are copies of $Z$. In fact, it is easy to see that if $I_{0} \neq B$, there is a monomial $u \notin I_{0}$ such that $x_{1} u, \cdots, x_{n} u \in I_{0}$, that $\left(I_{0}+u B\right) / I_{0} \cong Z$, and the result follows from Noetherian induction on $I_{0}$, since $I_{0}+B$ is a larger ideal of the same form.

Since 2) yields that $\operatorname{Tor}_{1}^{B}(Z, E)=0$, we see that for $I_{0}$ as above, $\operatorname{Tor}_{1}^{B}\left(B / I_{0}, E\right)=0$. Hence, since $0 \rightarrow I_{0} \rightarrow B \rightarrow B / I_{0} \rightarrow 0$ is exact, so is

$$
0 \rightarrow I_{0} \otimes_{B} E \rightarrow B \otimes_{B} E \rightarrow\left(B / I_{0}\right) \otimes_{B} E \rightarrow 0,
$$

and hence $I_{0} \otimes_{B} E \cong I_{0} E$. Thus, $I \otimes_{B} E \cong I E$ and $J \otimes_{B} E \cong J E$. Now we have the exact sequence:

$$
0 \rightarrow I \rightarrow J \rightarrow J / I \rightarrow 0,
$$

and $J / I \cong Z$. Applying $\otimes_{B} E$ and recalling $\operatorname{Tor}_{1}^{B}(Z, E)=0$, we have

$$
0 \rightarrow I E \rightarrow J E \rightarrow Z \otimes E \rightarrow 0
$$

is exact, and $Z \otimes_{B} E \cong E /\left(y_{1}, \cdots, y_{n}\right) E \neq 0$, by 1$)$, so that $I E \subseteq J E$. But then $I S \subseteq J S$, and so $\left(y_{1} \cdots y_{n}\right)^{k} \notin\left(y_{1}^{k+1}, \cdots, y_{n}^{k+1}\right)$. 
THEOREM 3. Let $R$ be a ring such that $R_{P}$ is a regular local ring for each maximal ideal $P$ of $R$, let $S$ be an extension ring such that $S$ is finitely presented as an $R$-module, and suppose that for each maximal ideal $M$ of $S$ and each system of parameters $y_{1}, \cdots, y_{n}$ of $M$, there is an S-module $E$ such that

$$
\left(y_{1}, \cdots, y_{n}\right) E \neq E
$$

$$
H_{1}\left(E ; y_{1}, \cdots, y_{n}\right)=0 \text {. }
$$

Then $R$ is a direct summand of $S$.

Proof. Let $P$ be a maximal ideal of $R$. It suffices to show that $R_{P}$ is a direct summand of $R_{P} \otimes S$ for each such $P$. Thus, we can reduce to the case where $R$ is local. Let $y_{1}, \cdots, y_{n}$ be a regular system of parameters for $R$. Since $S$ is module-finite over $R$, there is a maximal ideal $M$ of $S$ lying over $P$ such that $\operatorname{dim} S_{M}=\operatorname{dim} R_{P}$, and it follows that $y_{1}, \cdots, y_{n}$ is a system of parameters for $S_{M}$. Hence, by Proposition $4, y_{1}, \cdots, y_{n}$ have no relation like (\#) in $S_{M}$, and hence no such relation in $S$. The result now follows from Theorem 1.

Remark 4. Suppose that for each maximal ideal $M$ of $S$, the completion of $S_{M}$ possesses a Cohen-Macaulay module whose dimension is equal to that of $S_{M}$. Then this module certainly satisfies the requirements of Theorem 3. Moreover, it is not known whether every complete local ring possesses a maximal Cohen-Macaulay module (this is Conjecture $E$ of [6]). Thus, Conjecture $E$ implies Conjecture 1 here, and also implies that the restriction that $R$ contain a field can be removed from the hypothesis of Theorem 2.

Remark 5. Conjecture 1 is equivalent to a superficially stronger statement, Conjecture $1^{\circ}$ below. If $(a)=\left(a_{1}, \cdots, a_{n}\right)$ is an $n$-tuple of nonnegative integers and $x=\left(x_{1}, \cdots, x_{n}\right)$ is an $n$-tuple of elements of a $\operatorname{ring} S$, let $x^{(a)}=x_{1}^{a_{1}} \cdots x_{n}^{a_{n}}$. Write $(a) \geq(b)$ if $a_{i} \geq b_{i}, 1 \leq i \leq n$. Then:

CONJECTURE $1^{\circ}$. If $S$ is a local ring and $x_{1}, \cdots, x_{n}$ is a system of parameters, then $x^{(a)} \in\left(x^{\left(b_{t}\right)}: 1 \leq t \leq m\right) S$ if and only if for some $t$, (a) $\geq\left(b_{t}\right)$.

To see that Conjecture $1^{\circ}$ follows from Conjecture 1 , suppose $1^{\circ}$ fails and

$$
x^{(a)}=\sum_{t=1}^{n} s_{t} x^{\left(b_{t}\right)}, s_{t} \in S
$$


Choose $\left(a^{\prime}\right) \geq(a)$ with all entries of $\left(a^{\prime}\right)$ equal, say $\left(a^{\prime}\right)=(k, \cdots, k)$. If we multiply both sides of $(*)$ by $x^{\left(a^{\prime}\right)-(a)}$ we obtain a contradictory example in which the left hand side is $\left(x_{1} \cdots x_{n}\right)^{k}$. (For each $t,(a) \nsupseteq\left(b_{t}\right)$ implies that $\left.\left(a^{\prime}\right) \nsupseteq\left(b_{t}\right)+\left(a^{\prime}\right)-(a)\right)$. Hence, we can assume without loss of generality that $(a)=(k, \cdots, k)$. Now $(k, \cdots, k) \geq\left(b_{t}\right)$ implies that some entry of $b_{t}$ is at least $k+1$, and hence $\left(x^{\left(b_{t}\right)}: 1 \leq t \leq m\right) S \subset$ $\left(x_{1}^{k+1}, \cdots, x_{n}^{k+1}\right) S$, and $\left(x_{1} \cdots x_{n}\right)^{k} \in\left(x_{1}^{k+1}, \cdots, x_{n}^{k+1}\right) S$, contradicting Conjecture 1 .

Conjecture $1^{\circ}$ is known if the monomials involved all have the same degree [10], Theorem 21, p. 292. This result is used to prove the analytic independence of a system of parameters, and is really a somewhat stronger and more general statement. Hence, Conjecture 1 (or $1^{\circ}$ ) is quite a bit stronger than the analytic independence of a system of parameters.

Remark 6. Theorem 21 of [10] has a module-theoretic extension which is quite easy to prove. It is worth noting this extension, and also that the corresponding extension of Conjecture $1^{\circ}$ fails.

The module-theoretic extension of Theorem 21 of [10] is as follows: Let $x^{(a)}, x^{\left(b_{1}\right)}, \cdots, x^{\left(b_{m}\right)}$ be distinct monomials of the same degree $d$ in $a$ system of parameters $x_{1}, \cdots, x_{n}$ of a local ring $S$, and let $E$ be an $S$-module of dimension $n$, so that $x_{1}, \cdots, x_{n}$ is a system of parameters for $E$. Then

$$
x^{(a)} E \not \subset\left(x^{\left(b_{1}\right)}, \cdots, x^{\left(b_{m}\right)}\right) E .
$$

To prove this, let $N_{e}$ be the number of monomials of degree $e$ in $x_{1}, \cdots, x_{n}$. If $e \geq d$, then $\left(x_{1}, \cdots, x_{n}\right)^{e} E$ is $\subset\left(S_{e}\right) E$, where $S_{e}$ is the set of monomials of degree $e$ which are not multiples of $x^{(a)}$, so that $S_{e}$ has $N_{e}-N_{e-d}$ elements. Hence, $E_{e}=\left(x_{1}, \cdots, x_{n}\right)^{e} E /\left(x_{1}, \cdots, x_{n}\right)^{e+1} E$ has a filtration whose factors are $N_{e}-N_{e-d}$ homomorphic images of $E /\left(x_{1}, \cdots\right.$, $\left.x_{n}\right) E$, and $\ell\left(E_{e}\right) \leq b\left(N_{e}-N_{e-d}\right)$, where $b=\ell\left(E /\left(x_{1}, \cdots, x_{n}\right) E\right)$. It follows at once that the degree of the Hilbert polynomial $f(e)$ of $E$ (which agrees with $\ell\left(E /\left(x_{1}, \cdots, x_{n}\right)^{e} E\right)$ for large $\left.e\right)$ is equal to the degree of $N_{e}, n-1$, when it should have degree $n$.

To see that this does not generalize to the context of Conjecture $1^{\circ}$, note that if $\operatorname{dim} S \geq 2, x_{1}, x_{2}$ is part of a system of parameters, and $E$ is the ideal $\left(x_{1}^{2}, x_{2}^{2}\right) S$, then 


$$
x_{1}^{2} x_{2}^{2} E \subset\left(x_{1}^{3}, x_{2}^{3}\right) E
$$

Remark 7. Conjecture $E$ is known for local rings of dimension $\leq 2$. Hence, Conjectures 1 and $1^{\circ}$ hold in dimension $\leq 2$, and the hypothesis that $R$ contains a field can be dropped in Theorem 2 if $\operatorname{dim} R \leq 2$.

2. Ideally integrally closed domains. Call a domain $D$ ideally integrally closed (or $I I C$ ) if for every integral extension ring $S$ of $D$ and every ideal $I$ of $D$, IS $\cap D=I$. Thus, every ideal of $D$ is contracted from every integral extension. In this section we briefly explore this notion. We begin with some rather straight forward observations:

A. An IIC domain $D$ is integrally closed. (For if $b / a$ is in the integral closure $S$ of $D, b$ is in $(a D) R \cap D=a D$, and $b / a$ is in $D$.)

B. To determine whether $D$ is IIC it suffices to consider only domains $S$ integral over $D$. Hence, it suffices to consider the integral closure $S$ of $D$ in an algebraic closure of the fraction field of $D$. (For if $I S \cap$ $D=J$, and $J$ properly contains $I$, we can choose a prime $P$ of $S$ maximal with respect to disjointness from the multiplicative system $D-\{0\}$, and then $I(S / P) \cap D$ contains $J$.)

C. It suffices to consider only module-finite domain extensions of $D$, since each domain integral over $D$ is a directed union of these. Likewise, it suffices to consider only finitely generated ideals of $D$. Hence, Prüfer domains are IIC.

D. If $D$ is IIC, then so is $T^{-1} D$ for any multiplicative system $T$ in $D$. (For if $S$ is integral over $T^{-1} D$ and $I$ is an ideal of $T^{-1} D$ such that $I S \cap T^{-1} D=J$ properly contains $I$, then if $S_{0}$ is the integral closure of $D$ in $S$, it is easy to see that $\left((I \cap D) S_{0}\right) \cap D=J \cap D$ properly contains $I \cap D$.

E. If for each maximal ideal $M$ of the domain $D$ the domain $D_{M}$ is $I I C$, then $D$ is IIC. (For suppose $S$ is a domain integral over $D$ and $I R \cap D=J$ properly contains $I$. Choose a maximal ideal $M$ of $D$ which contains $I: J$ in $D$. $\left(\left(I D_{M}\right)(D-M)^{-1} S\right) \cap D_{M}$ contains $J D_{M}$ which properly contains $I D_{M}$.)

F. If $D$ is a domain which is a directed union of IIC domains, then $D$ is an IIC domain. In fact, it suffices that each subdomain of $D$ 
which is finitely generated over the prime subdomain $D_{0}$ of $D$ be contained in a subdomain of $D$ which is IIC. (For suppose $j \notin\left(i_{1}, \cdots, i_{n}\right) D$ but $S$ is a domain integral over $D$ such that $J=\sum_{t} s_{t} i_{t}, s_{t} \in S$ for each $t$. Clearly, we can choose a ring $D_{1} \subset D$ finitely generated over $D_{0}$ which contains $j, i_{1}, \cdots, i_{n}$ and sufficiently many elements so that $r_{1}, \cdots, r_{n}$ are integral over $D_{1}$. Then we can choose an IIC subdomain $D_{2}$ of $D$ with $D_{1} \subset D_{2}$. Let $R_{2}=D_{2}\left[r_{1}, \cdots, r_{n}\right]$. Then $j \notin\left(i_{1}, \cdots, i_{n}\right) D_{2}$, but $j \in\left(i_{1}\right.$, $\left.\cdots, i_{n}\right) R_{2}$, contradiction.)

G. If $D$ is a domain such that every module-finite extension domain $S$ of $D$ has $D$ as a direct summand (as a D-module), then $D$ is IIC. (This is immediate from Proposition 1.)

H. If $D$ is a domain which contains the rationals, $Q$, then $D$ is IIC if and only if $D$ is integrally closed. (This is immediate from A., G., above, and Lemma 2.)

I. If $D \subset R, R$ is an IIC domain, and for every ideal $I$ of $D$, $I R \cap D=I$, then $D$ is IIC. In particular, if $R$ is IIC and faithfully flat over $D$, or if $R$ is IIC and $D$ is a direct summand of $R$, then $D$ is IIC. It follows that if the completion $D$ of a Noetherian local domain $D$ is an IIC domain, then $D$ is an IIC domain. (Let $L$ be an algebraically closed field which contains $R$. If $C$ is a domain integral over $D$, there is a copy of $C$ in $L$, and $R[C]$ is a domain integral over $R$. Hence, for any ideal $I$ of $D$, we have $I C \cap D \subset(I R[C]) \cap(R \cap D)=(I R[C] \cap R)$ $\cap D=(I R) \cap D=I$.)

Let us say that a proper ideal $I$ of a commutative ring $R$ is big if it is not an intersection of properly larger ideals. Thus, $I$ is big if and only if there exists $x \notin I$ such that $x$ is in every ideal properly larger than $I$. Obviously, $I$ is big if and only if (0) is big in $R / I$. By a trivial application of Zorn's lemma, every proper ideal of a commutative ring is an intersection of big ideals. Hence:

J. A domain $D$ is IIC if and only if for every module-finite domain extension $S$ of $D$ and every big ideal $I$ of $D, I S \cap D=I$.

It is easy to show that if $R$ is Noetherian then $I \subseteq R$ is big if and only if $I$ is primary to a maximal ideal and irreducible. Thus, (0) is big in $R$ if and only if $R$ is a zero-dimensional Gorenstein local ring. 
K. A Gorenstein local domain $(D, P)$ is IIC if and only if for every module-finite domain extension $S$ of $D, D$ is a direct summand of $S$. Moreover, if $\left\{I_{k}\right\}$ is a sequence of P-primary irreducible ideals of $D$ cofinal with $\left\{P^{k}\right\}$, then $D$ is IIC if and only if for every module-finite domain extension $S$ of $D$, each $I_{k}$ is contracted. (It suffices to show that if each $I_{k}$ is contracted from $S$ then $D$ is a direct summand of $S$. See the second paragraph of Remark 2.)

L. Let $(D, P)$ be a two-dimensional Gorenstein local domain and let $x_{1}, x_{2}$ be a system of parameters. If $\left(x_{1}, x_{2}\right)$ is contracted from every module-finite extension domain $S$ of $D$, then $D$ is IIC. (Let $u$ generate $\left(x_{1}, x_{2}\right): P$ modulo $\left(x_{1}, x_{2}\right)$. If suffices to show that we cannot have $x_{1}^{k} x_{2}^{k} u=s_{1} x_{1}{ }^{k+1}+s_{2} x_{2}{ }^{k+1}$. Let $S^{\prime}$ be the integral closure of $S$. Then $S^{\prime}$ is Noetherian [8], Theorem (33.12), p. 120, and semilocal, and $x_{2}$ is not a zerodivisor modulo $x_{1} S^{\prime}$, for $S^{\prime}$ is integrally closed and $\operatorname{dim} S^{\prime}=2$, so that $S^{\prime}$ is Cohen-Macaulay. Since $x_{1}, x_{2}$ is an $S^{\prime}$-sequence, we must have that $x_{1}^{k}$ divides $s_{2}$ in $S^{\prime}$, and that $x_{2}^{k}$ divides $s_{1}$ in $S^{\prime}$. Hence, $x_{1}^{k} x_{2}^{k} u=x_{1}^{k+1} x_{2}^{k} s_{1}^{\prime}+x_{1}^{k} x_{2}^{k+1} s_{2}^{\prime}, s_{1}^{\prime}, s_{2}^{\prime} \in S^{\prime}$, and $u=x_{1} s_{1}^{\prime}+x_{2} s_{2}^{\prime}$, so that $\left(x_{1}, x_{2}\right) D$ is not contracted from $D\left[s_{1}^{\prime}, s_{2}^{\prime}\right]$, a contradiction.)

This completes our list of observations about IIC domains. A somewhat more interesting result (although the real work was done in the proof of Theorem 2) is:

Proposition 5. Let $D$ be a domain such that for each maximal ideal $M$ of $D, D_{M}$ is a regular local ring, and suppose that $D$ contains a field. Then $D$ is $I I C$.

Proof. This follows at once from observations E. and G. and Theorem 2.

The following proposition shows that there are interesting nonregular examples of $I I C$ domains in characteristic $p$.

Proposition 6. Let $k$ be any field and let $x_{1}, \cdots, x_{n}$ be indeterminates over $k$. Let $\mathfrak{m}$ be a set of monomials (expressions of the form $\left.x_{1}^{t_{1}} \cdots x_{n}^{t_{n}}\right)$ in $x_{1}, \cdots, x_{n}$ such that the subring $D=k[\mathfrak{m}] \subset k\left[x_{1}, \cdots, x_{n}\right]$ is integrally closed (in the fraction field of $D$ ). Then $D$ is IIC.

Proof. We use the results of [5]. By Proposition 1 of [5], the fact that $k[\mathrm{~m}]$ is normal implies that $\mathfrak{m}$ generates a normal semigroup 
$\mathbb{S}$ of monomials and hence that $\mathbb{S}$ is isomorphic to a full semigroup $\mathfrak{I}$ of monomials (possibly in different indeterminates). But then $k[\mathfrak{T}]$ is a direct summand of a polynomial ring, by Lemma 1 of [5], and it follows from Theorem 2 here and observation $\mathrm{I}$. that $k[\mathrm{~m}] \cong k[\mathfrak{T}]$ is $I I C$.

The rest of this paper is devoted primarily to a discussion of open questions and examples.

Example 1, revisited. The ring $D$ of Example 1 is an integrally closed Gorenstein ring which is not IIC. $D$ has, moreover, a modulefinite extension which is $I I C$.

QUESTION 1. Is there some nice class of domains which have minimal or minimum IIC extensions (analogues of integral closures)?

In connection with this we note that the intersection of a decreasing sequence of $I I C$ domains need not be $I I C$. In Example 1, $D$ is countable, and hence has only countably many height 1 primes: say $P_{1}, P_{2}, P_{3}, \cdots$ is an enumeration of them. Let $T_{i}=D-\left(\bigcup_{t=1}^{i} P_{t}\right)$ and let $D_{i}=T_{i}^{-1} D$. Then $D_{i}$ is an integrally closed semilocal domain of Krull dimension 1, and hence $D_{i}$ is $I I C$ by Remark 7 . But $D=\bigcap_{i} D_{i}$.

However, I do not know the answer to

Question 2. If $D_{1}, D_{2}$ are $I I C$ subdomains of $D$, is $D_{1} \cap D_{2} I I C$ ?

Some other obvious lines of inquiry are:

Question 3. If $D$ is $I I C$, is $D[x] I I C$ ? Is $D[[x]] I I C ?$ Here, $x$ is an indeterminate.

QUESTION 4. If $D$ is finitely generated over a field, is

$$
\left\{P \in \operatorname{Spec} D: D_{P} \text { is } I I C\right\}
$$

Zariski open in Spec $D$ ?

QUESTION 5. If $D_{1}$ and $D_{2}$ are $I I C$ domains finitely generated over an algebraically closed field $k$, is $D_{1} \otimes_{k} D_{2} I I C$ ? (If $D_{2}=k[x]$, Question 5 becomes a special case of Question 3.)

EXAMPLE 2. An interesting example of UFD which is not $I I C$ comes out of invariant theory. We first need:

Proposition 7. If $D$ is local and IIC and $R$ is a module-finite equidimensional extension of $D$ which is Cohen-Macaulay, then $D$ is Cohen-Macaulay. 
Proof. Let $x_{1}, \cdots, x_{n}$ be a system of parameters for $D$. It follows easily that $x_{1}, \cdots, x_{n}$ is an $R$-sequence in $R$. But then, since $D$ is $I I C$, $x_{1}, \cdots, x_{n}$ is an $R$-sequence in $D$, for if $d x_{k+1} \in\left(x_{1}, \cdots, x_{k}\right) D$, then $d \in$ $\left(x_{1}, \cdots, x_{k}\right) R \cap D=\left(x_{1}, \cdots, x_{k}\right) D$.

Now let $K$ be a field of characteristic 2 and let $R_{1}=K\left[x_{1}, x_{2}, x_{3}, x_{4}\right]$. Let $g$ be the $K$-automorphism of $R_{1}$ such that $g\left(x_{4}\right)=x_{4}$ while $g\left(x_{i}\right)=$ $x_{i}+x_{i+1}, i=1,2,3$. Let $G$ be the cyclic group of order 4 generated by $g$, so that we have an action of $G$ on $R_{1}$. The ring of invariants $D_{1}$ is a graded $K$-algebra which is known [1] to be a non-Cohen-Macaulay $U F D$ of dimension 4 . Let $D, R$ by the localizations of $D_{1}, R_{1}$ at their respective irrelevant maximal ideals. Since $R$ is module-finite over $D$ and Cohen-Macaulay (in fact, regular) while $D$ is not Cohen-Macaulay, it follows from Proposition 7 that $D$ is not $I I C$.

On the other hand we note:

Proposition 8. If $G$ is a finite group acting on an IIC domain $D$ and the order $d$ of $G$ is invertible in $D$, then the ring of invariants

$$
D^{G}=\{r \in D: g(r)=r \text { for all } g \in G\}
$$

is $I I C$.

Proof. The map $h: D \rightarrow D^{G}$ by

$$
h(r)=(1 / d) \sum_{g \in G} g(r)
$$

is a $D^{G}$-module retraction of $D$ onto $D^{G}$.

Example 3. Proposition 6 provides a number of interesting examples of non-regular $I I C$ domains in characteristic $p>0$. One example is the usual homogeneous coordinate ring for a multiprojective space

$$
\boldsymbol{P}^{t_{1}} \times \cdots \times \boldsymbol{P}^{t_{n}}
$$

namely, the Segre product (see [2] and [7], $\S 13$ ) of $n$ polynomial rings in $t_{1}+1, \cdots, t_{n}+1$ variables, respectively. A particularly simple case is $\boldsymbol{P}^{1} \times \boldsymbol{P}^{1}$ where the homogeneous coordinate ring is

$$
\begin{aligned}
D & =k\left[x_{1} y_{1}, x_{1} y_{2}, x_{2} y_{1}, x_{2} y_{2}\right] \\
& \cong k[p, q, r, s] /(p s-q r)
\end{aligned}
$$

Another example is the homogeneous coordinate ring for $\boldsymbol{P}^{n-1}$ spanned 
by all forms whose degree is a multiple of $d$ in $k\left[x_{1}, \cdots, x_{n}\right]$. The simplest case is

$$
D=k\left[x^{2}, x y, y^{2}\right] \cong k[p, q, r] /\left(p r-q^{2}\right)
$$

Finally, we note that some of the examples above have an invarianttheoretic flavor, and we raise the following question:

QUESTION 6. If $k$ is an algebraically closed field of characteristic $p$ and $G$ is a connected linear algebraic group acting rationally on an $n$-dimensional vector space $V$, so that the symmetric algebra $R=S(V)$ $\cong k\left[x_{1}, \cdots, x_{n}\right]$, then is the ring of invariants $R^{G}$ of the induced action of $G$ on $R$ IIC? More generally, if $R$ is an IIC domain and $D$ is integrally closed in $R$, is $D I I C$ ?

\section{REFERENCES}

[1] $M^{\text {me }} M$. Bertin, Anneaux d'invariants d'anneaux de polynomes, en caracterisque p. Comptes Rendus. Ser. A. $264: 2$ (1967), 653-656.

[2] W. L. Chow, On unmixedness theorem, Amer. J. Math. 86 (1964), 799-822.

[ 3 ] J. A. Eagon and M. Hochster, R-sequences and indeterminates, preprint.

[ 4 ] A. Grothendieck (notes by R. Hartshorne), Local cohomology, Springer-Verlag Lecture Notes in Mathematics No. 41, 1967.

[5] M. Hochster, Rings of invariants of tori, Cohen-Macaulay rings generated by monomials, and polytopes, Annals of Math., 96 (1972), 318-337.

[6] M. Hochster, Cohen-Macaulay modules, Proc. Kansas Commutative Algebra Conference, Springer-Verlag, Lecture Notes in Mathematics No. 311, 1973.

[ 7 ] M. Hochster and J. A. Eagon, Cohen-Macaulay rings, invariant theory, and the generic perfection of determinantal loci, Amer. J. Math. 93 (1971), 1020-1058.

[ 8 ] M. Nagata, Local rings, Interscience, New York, 1962.

[ 9 ] D. Taylor, Ideals generated by monomials in an R-sequence, Thesis, University of Chicago, 1966.

[10] O. Zariski and P. Samuel, Commutative algebra, Vol. II, Van Nostrand, Princeton, 1960.

University of Minnesota 